\title{
Effect of Melatonin on Clinical Outcome of Patients with Repeated Cycles after Failed Cycles of In Vitro Fertilization and Intracytoplasmic Sperm Injection
}

\section{Qi Zhu}

Department of Biomedical Engineering, Anhui Medical University, Hefei, China.

\section{Kaijuan Wang}

Reproductive Medicine Center, Depatment of Obstetrics and Gynecology, the First Affiliated Hospital of Anhui Medical University, Hefei, China.

\section{Chao Zhang}

Reproductive Medicine Center, Depatment of Obstetrics and Gynecology, the First Affiliated Hospital of Anhui Medical University, Hefei, China.

\section{Beili Chen}

Reproductive Medicine Center, Department of Obstetrics and Gynecology,the First Affiliated Hospital of Anhui Medical University, Hefei, China

\section{Huijuan Zou}

Reproductive Medicine Center, Department of Obstetrics and Gynecology, the First Affiliated Hospital of Anhui Medical University, Hefei, China.

\section{Weiwei Zou}

Reproductive Medicine Center, Department of Obstetrics and Gynecology, the First Affiliated Hospital of Anhui Medical University, Hefei, China.

\section{Rufeng Xue}

Reproductive Medicine Center, Department of Obstetrics and Gynecology, the First Affiliated Hospital of Anhui Medical University, Hefei, China

\section{Dongmei Ji}

Reproductive Medicine Center, Department of Obstetrics and Gynecology, the First Affiliated Hospital of Anhui Medical University, Hefei, China.

\section{Zhaojuan Yu}

Reproductive Medicine Center, Department of Obstetrics and Gynecology, the First Affiliated Hospital of Anhui Medical University, Hefei, China.

\section{Bihua Rao}

Reproductive Medicine Center, Department of Obstetrics and Gynecology, the First Affiliated Hospital of Anhui Medical University, Hefei, China.

\section{Yunxia Cao}


Reproductive Medicine Center, Department of Obstetrics and Gynecology, the First Affiliated Hospital of Anhui Medical University, Hefei, China.

\section{Ding Ding}

Reproductive Medicine Center, Department of Obstetrics and Gynecology, the First Affiliated Hospital of Anhui Medical University, Hefei China.

\section{Zhiguo Zhang ( $\nabla$ zzg_100@163.com )}

Anhui Medical University https://orcid.org/0000-0003-1483-3321

\section{Research Article}

Keywords: melatonin, embryo cultures in vitro, failed IVF/ICSI cycles, embryonic development.

Posted Date: May 6th, 2021

DOl: https://doi.org/10.21203/rs.3.rs-443250/v1

License: (c) (i) This work is licensed under a Creative Commons Attribution 4.0 International License. Read Full License

Version of Record: A version of this preprint was published at Zygote on February 28th, 2022. See the published version at https://doi.org/10.1017/S0967199421000770. 


\section{Abstract}

Background: Melatonin (MT), as a powerful antioxidant, can effectively ameliorate the in vitro development of animal embryos, but few studies have been made on human embryos. Therefore, we investigated whether the application of MT in embryo cultures can improve embryonic development and clinical outcomes in patients with repeated cycles after failed in vitro fertilization/intracytoplasmic sperm injection (IVF/ICSI) cycles.

Methods: Human immature oocytes from controlled ovarian hyperstimulation cycles were collected for in vitro maturation culture and ICSI fertilization. The obtained embryos were cultured in vitro in medium containing $0,10^{-11}, 10^{-9}, 10^{-7}$ or $10^{-5} \mathrm{M} \mathrm{MT}$, and $10^{-9} \mathrm{M}$ was determined to be the optimal concentration. Subsequently, 140 patients who experienced failed IVF/ICSI cycles received 140 cycles of embryo culture in vitro with medium containing $10^{-9} \mathrm{M} \mathrm{MT}$. High-quality blastocysts were collected and cryopreserved for three months before vitrified-warmed embryo transfer. These culture cycles served as the experimental $\left(10^{-9} \mathrm{M}\right)$ group. The control $(0 \mathrm{M})$ group comprised previous failed cycles. The patients were further divided into subgroups of 1,2 or $\geq 3$ failed cycles. The fertilization and embryo development status were compared.

Results: The fertilization, cleavage, high-quality embryo, blastocyst, and high-quality blastocyst rates of the $10^{-9} \mathrm{M}$ group were significantly higher than $0 \mathrm{M}(87.7 \%$ vs. $83.6 \%, p<0.01 ; 94.1 \%$ vs. $90.5 \%, p<0.01$; $58.3 \%$ vs. $43.8 \%, 51.1 \%$ vs. $41.8 \%, 43.4 \%$ vs. $22.9 \%$, all $p<0.0001$ ). A total of 50 vitrified-warmed cycle transfers were performed. A clinical pregnancy rate of $40 \%$ was obtained.

Conclusion: The application of MT to embryo cultures in vitro improved embryonic development in patients with repeated cycles after failed IVF/ICSI cycles and obtained good clinical outcomes. The optimal concentration of MT was $10^{-9} \mathrm{M}$.

Trial registration: Name of the registry: Effect of melatonin on clinical outcome of patients with repeated cycles after failed cycles of in vitro fertilization and intracytoplasmic sperm injection; registration number: ChiCTR2100045552; date of registration: April 19, 2021(Retrospectively registered); URL of trial registry record: www.medresman.org.cn.

\section{Background}

Assisted reproductive technology (ART) involves the use of medical methods to artificially manipulate oocytes, sperm, fertilized eggs, and embryos to achieve the goal of conception [1]. Among them, in vitro fertilization (IVF) and intracytoplasmic sperm injection (ICSI) are the core technologies of ART. IVF refers to the natural fertilization of eggs and sperm through artificial methods, and the subsequent in vitro culture of early embryos, while ICSI refers to the process of injecting a single sperm into the cytoplasm of an egg using micromanipulation technology to achieve fertilization. In vitro fertilization and embryo transfer (IVF-ET) technology has become an important means of infertility treatment since its discovery, 
but the risk of poor embryo development with the traditional IVF/ICSI treatment cycle is still unavoidable [2], which leads to pregnancy failure due to poor development of the embryos transferred. Therefore, exploring an efficient technology of embryo culture in vitro to obtain high-quality embryos is one of the key features to improve the effect of ART treatment.

Melatonin (MT) is a multifunctional molecule that is mainly produced in the pineal gland [3]. It is a powerful free radical scavenger and antioxidant [4]. MT and its metabolites can directly remove reactive oxygen species (ROS) in cells, activate antioxidant enzymes, increase the expression level and activity of glutathione (GSH), and inhibit pro-oxidant enzymes to reduce cell oxidation injury, which can improve oocyte in vitro maturation (IVM) and embryonic development [5]. It has been reported that adding the appropriate amount of MT to IVM and embryo culture medium can significantly improve the developmental potential of oocytes and the in vitro development of embryos [6-8]. Through research on human IVF and IVM, Li et al. found that the addition of MT to the embryo culture medium can improve the clinical outcomes of IVF and IVM [9]. In 2019, our research team added MT to the IVM medium to culture human immature oocytes from a controlled ovarian hyperstimulation $(\mathrm{COH})$ cycle, which significantly reduced the levels of ROS and $\mathrm{Ca}^{2+}$ in oocytes during the IVM process, increased mitochondrial membrane potential, and improved embryo development, which eventually resulted in healthy offspring [10]. The present study was the first attempt to use embryo culture medium supplemented with MT to culture embryos of patients undergoing repeated cycles after failed IVF/ICSI cycles, and aimed to explore whether the application of MT could improve the embryonic development and clinical outcomes of patients with repeated IVF/ICSI cycles.

\section{Materials And Methods}

\section{Research patients}

A total of 140 patients with failed cycles who visited the Reproductive Medicine Center of the First Affiliated Hospital of Anhui Medical University from June 2020 to November 2020 were selected. Inclusion criteria: (1) at least one IVF/ICSI failed cycle;(2) age of patient $\leq 36$ years. Excluding genetic factors of both men and women, the causes of infertility included 18 cases of male-only factors, involving oligoasthenoteratozoospermia; 77 cases of female-only factors, involving 41 cases of sequelae of pelvic inflammatory disease, 26 cases of polycystic ovary syndrome, and 10 cases of endometriosis; 40 cases of combined male and female factors; and 5 cases with unknown causes. Before conducting the study, the partners of all enrolled patients were interviewed and provided signed informed consent.

\section{$\mathrm{COH}$ regimen}

All patients in this study were treated with gonadotropin hormone-releasing hormone $(\mathrm{GnRH})$ antagonist (flexible regimen) for ovarian stimulation. Recombinant human follicle stimulating hormone (Gn, Gonal F; Serino Barueri, SP, Brazil) was given on the second or third day of the menstrual cycle for ovarian hyperstimulation. After four to five days, the dosage of $\mathrm{Gn}$ was adjusted according to the follicle size and hormone level. When the dominant follicle reached 12-14 mm, GnRH antagonist (GnRH-A, Cetrotide, 
Merck Serono SA - Geneva, Switzerland) was added until the day of the human chorionic gonadotropin (HCG) injection. When there were two or three follicles with a diameter $\geq 18 \mathrm{~mm}, \mathrm{HCG}(10,000 \mathrm{U}$; Pregnyl; AESCA Pharma, Austria) was injected, and oocyte retrieval was completed after $36-38 \mathrm{~h}$ under the guidance of a transvaginal ultrasound.

\section{Experimental design}

\section{Experiment 1}

Human immature oocytes obtained from the $\mathrm{COH}$ cycle were collected for IVM to obtain mature oocytes (IVM-MII) in vitro and perform ICSI insemination. Then, embryos were cultured in vitro in medium containing $0,10^{-11}, 10^{-9}, 10^{-7}$ or $10^{-5} \mathrm{M} \mathrm{MT}$. Ultimately, $10^{-9} \mathrm{M}$ was determined to be the optimal concentration of MT.

\section{Experiment 2}

For the 140 enrolled patients with failed IVF/ICSI cycles, the embryo culture medium containing $10^{-9} \mathrm{M}$ MT was prepared to conduct embryo culture in vitro in the subsequent 140 repeated cycles. High-quality blastocysts were collected and cryopreserved. These 140 MT culture cycles were designated as the experimental group (10-9 $\mathrm{M}$ group), and the control group consisted of the previous failed cycles of the same patients ( $0 \mathrm{M}$ group). In addition, according to the number of failed cycles, the patients were further divided into subgroups of one, two, or at least three failed cycles, and the fertilization and embryo development status of each subgroup in the $10^{-9} \mathrm{M}$ and $0 \mathrm{M}$ groups were compared and analyzed.

\section{Experiment 3}

After 3 months, vitried-warmed embryo transfer was performed. This study was discussed and approved by the Reproductive Ethics Committee of the First Affiliated Hospital of Anhui Medical University. The experimental process is shown in Fig. 1.

\section{IVM of oocytes from $\mathrm{COH}$ cycle}

Human immature oocytes with normal morphology from the $\mathrm{COH}$ cycle were collected and placed into IVM medium prepared and balanced overnight to culture for $24 \mathrm{~h}$. Subsequently, the IVM-MII oocytes were selected for ICSI insemination, followed by five or six days of embryo culture in vitro. The detailed process has been previously reported in published literature [11].

\section{Preparation of the embryo culture medium}

An appropriate amount of embryo culture medium (cleavage or blastocyst; Cook, Sydney, Australia) was prepared, and then an appropriate concentration of MT was added. Next, six to eight droplets (30 $\mu \mathrm{l} / \mathrm{drop})$ were made in a dish with a diameter of $60 \mathrm{~mm}$, and were covered with an appropriate amount of tissue culture oil. Finally, the dish was placed at $37^{\circ} \mathrm{C}$ with $6 \% \mathrm{CO}_{2}$ and saturated humidity for $18 \mathrm{~h}$ of equilibration. 


\section{ICSI/IVF insemination}

Under a microscope, the cumulus-oocyte complex (COC) in follicular fluid was picked up and placed into balanced fertilization medium (Cook, Sydney, Australia) at $37^{\circ} \mathrm{C}$ with $6 \% \mathrm{CO}_{2}$ and saturated humidity for 4-6 h of culture in vitro, followed by ICSI or IVF insemination. For ICSI insemination, its process has been described in detail in our previously published literature [12]. Following ICSI, the inseminated oocytes were directly transferred into the cleavage culture medium containing MT for embryo culture in vitro. For IVF insemination, the $\mathrm{COC}$, which experienced $4-6 \mathrm{~h}$ of culture in vitro, was transferred into the fertilization medium containing $5 \times 10^{5} / \mathrm{ml}$ of grade $A$ and grade $B$ sperm for $6 \mathrm{~h}$ of culture at $37^{\circ} \mathrm{C}$ with $6 \% \mathrm{CO}_{2}$ and saturated humidity. Next, the granule cells around the oocytes were removed and those with the second polar body, which were considered fertilized oocytes, were selected under an inverted microscope. Finally, all fertilized oocytes were transferred into cleavage culture medium with MT for subsequent embryo culture.

\section{Embryo culture}

The oocytes undergoing in vitro culture in the balanced cleavage culture droplets (one oocyte/drop) were observed based on their fertilization status 14-16 $\mathrm{h}$ after insemination, and then the culture was continued under $37^{\circ} \mathrm{C}, 6 \% \mathrm{CO}_{2}$, and saturated humidity. Two days later, all of the formed cleavage embryos were transferred into balanced blastocyte culture droplets (one to three embryos/drop) for additional two or three days of blastocyst culture. Finally, the obtained high-quality blastocysts (see Fig. 2) were selected and cryopreserved in $-196^{\circ} \mathrm{C}$ liquid nitrogen using the vitrification method. In this process, all embryos formed were scored according to Tomas [13]and Gardner [14]scoring standards.

\section{Embryo transfer and pregnancy determination}

Three months later, the cryopreserved blastocyst was warmed. According to the patient's age, one or two warmed blastocysts were transferred into the uterus under B-ultrasound guidance. Two weeks after the embryo transfer, serum hCG levels were examined and a biochemical pregnancy was defined as a positive $h C G$ value ( $\geq 25 \mathrm{IU} / \mathrm{L}$ ). At seven weeks following embryo transfer, the presence of a gestational sac identified by an ultrasound scan was referred to as a clinical pregnancy. For the detailed operation process of embryo vitrification and warming, please refer to our previously published literature [15].

\section{Statistical analysis}

SPSS software version 23.0 was used to perform statistical analysis. The differences in means between continuous variables [female age, male age, basic follicle-stimulating hormone (FSH) level, basic luteinizing hormone (LH) level, basic E2 level, duration infertility, average no.oocyte retrieval and body mass index $(\mathrm{BMI})$ ] were expressed as mean $\pm \mathrm{SD}$. The categorical data from the developmental competence (rates of fertilization, cleavage, high-quality cleavage embryo, and high-quality blastocyst) in each group were analyzed using chi-squared test or Fisher's exact test. $P$-values $<0.05$ were considered statistically significant. 


\section{Results}

\section{Screening the optimal MT concentration for embryo culture medium}

A total of 400 human immature oocytes of $198 \mathrm{COH}$ cycles from 198 patients were collected for IVM culture, and 295 IVM-MII oocytes were obtained. ICSI insemination and embryo culture in vitro were performed, and the detailed results are shown in Table 1. There were no significant differences in age, FSH, luteinizing hormone (LH), estradiol (E2), body mass index (BMI), years of infertility, or number of oocytes obtained in each group (see Table 1).

Table 1

Comparison of baseline data of the 5 groups of patients

\begin{tabular}{|lllllll|}
\hline & OM & $10^{-11} \mathbf{M}$ & $10^{-9} \mathbf{M}$ & $10^{-7} \mathbf{M}$ & $1^{-5} \mathbf{M}$ & $\begin{array}{c}P \text { - } \\
\text { value }\end{array}$ \\
\hline Male age (yr) & $31.18 \pm$ & $30.85 \pm$ & $30.95 \pm$ & $30.89 \pm$ & $30.24 \pm$ & $>0.05$ \\
& 3.343 & 3.890 & 4.214 & 3.748 & 3.494 & \\
\hline Female age (yr) & $30.15 \pm$ & $29.33 \pm$ & $29.79 \pm$ & $30.14 \pm$ & $29.17 \pm$ & $>0.05$ \\
& 3.549 & 2.787 & 3.361 & 2.987 & 3.011 & \\
Infertility period (yr) & $3.200 \pm$ & $3.074 \pm$ & $3.000 \pm$ & $3.000 \pm$ & $3.057 \pm$ & $>0.05$ \\
& 1.800 & 1.796 & 1.593 & 1.493 & 1.598 & \\
No. of retrieved & $18.68 \pm$ & $18.44 \pm$ & $18.00 \pm$ & $18.67 \pm$ & $19.13 \pm$ & $>0.05$ \\
oocytes & 8.404 & 5.366 & 7.191 & 6.533 & 7.932 & \\
FSH & $6.663 \pm$ & $7.034 \pm$ & $7.111 \pm$ & $6.465 \pm$ & $7.055 \pm$ & $>0.05$ \\
& 2.259 & 1.911 & 1.428 & 1.901 & 2.362 & \\
LH & $5.700 \pm$ & $4.959 \pm$ & $5.721 \pm$ & $5.334 \pm$ & $4.965 \pm$ & $>0.05$ \\
& 2.679 & 2.582 & 5.465 & 3.286 & 3.158 & \\
E2 & $167.9 \pm$ & $178.0 \pm$ & $162.1 \pm$ & $177.7 \pm$ & $163.2 \pm$ & $>0.05$ \\
& 100.4 & 94.5 & 96.43 & 109.5 & 94.59 & \\
\hline BMI & $21.76 \pm$ & $21.66 \pm$ & $22.76 \pm$ & $21.76 \pm$ & $21.91 \pm$ & $>0.05$ \\
& 2.791 & 1.920 & 3.680 & 2.380 & 3.117 &
\end{tabular}

Values are presented as mean \pm standard deviation.

As shown in Table 2, after adding $10^{-9} \mathrm{M}$ MT to the embryo culture medium, the fertilization, cleavage, blastocyst and high-quality blastocyst rates of the $10^{-9} \mathrm{M}$ group were significantly different compared to those of the $0 \mathrm{M}$ group $(p<0.01)$. The blastocyst and high-quality blastocyst rates of the $10^{-9} \mathrm{M}$ group were significantly different compared to the $10^{-5} \mathrm{M}$ group $(p<0.01, p<0.001)$, and the blastocyst rate of the $10^{-9} \mathrm{M}$ group was significantly different from that of the $10^{-11} \mathrm{M}$ group $(p<0.01)$. The above data 
revealed that the addition of $10^{-9} \mathrm{M} \mathrm{MT}$ to the embryo culture could significantly improve the fertilization and in vitro development of the fertilized embryo, and $10^{-9} \mathrm{M}$ was the optimal concentration of MT.

Table 2

The effects of different concentrations of MT on embryo development in vitro

\begin{tabular}{|c|c|c|c|c|c|}
\hline & $\mathrm{OM}$ & $10^{-5} \mathrm{M}$ & $10^{-7} \mathrm{M}$ & $10^{-9} \mathrm{M}$ & $10^{-11} \mathrm{M}$ \\
\hline IVM-MII (n) & 61 & 58 & 59 & 61 & 56 \\
\hline Fertilization & 85.2 & 87.9 & 83.1 & 90.2 & 78.6 \\
\hline rate (\%) & $(52 / 61)$ & $(51 / 58)$ & $(49 / 59)$ & $(55 / 61)^{a}$ & $(44 / 56)$ \\
\hline Cleavage & 82.7 & 88.2 & 85.7 & 98.2 & 84.1 \\
\hline rate $(\%)$ & $(43 / 52)$ & $(45 / 51)$ & $(42 / 49)$ & $(54 / 55)^{a}$ & $(37 / 44)$ \\
\hline Blastocyst & 16.3 & 11.1 & 21.4 & 42.6 & 16.2 \\
\hline rate $(\%)$ & $(7 / 43)$ & $(5 / 45)$ & $(9 / 42)$ & $(23 / 54)^{a, c, d}$ & $(6 / 37)$ \\
\hline \multirow[t]{2}{*}{ High-quality blastocyst rate (\%) } & 4.7 & 4.4 & 9.5 & 24.1 & 2.7 \\
\hline & $(2 / 43)$ & $(2 / 45)$ & $(4 / 42)$ & $(13 / 54)^{a, b, d}$ & $(1 / 37)$ \\
\hline \multicolumn{6}{|c|}{$\begin{array}{l}\text { Datas analyzed using Chi-square or Fisher Exact test. Different symbols within columns and different } \\
\text { letters within columns and within rows indicate significant differences. }{ }^{\mathrm{a}} P<0.01 \text {, compared with } 0 \mathrm{M} \\
\text { group ; }{ }^{\mathrm{b}} P<0.01 \text {, compared with } 10^{-5} \mathrm{M} \text { group ; }{ }^{\mathrm{C}} P<0.001 \text {, compared with } 10^{-5} \mathrm{M} \text { group ; }{ }^{\mathrm{d}} P<0.01 \text {, } \\
\text { compared with } 10^{-11} \mathrm{M} \text { group. }\end{array}$} \\
\hline
\end{tabular}

\section{Fertilization and subsequent embryo development of oocytes in the $10^{-9} \mathrm{M}$ and $0 \mathrm{M}$ groups}

Table 3 showed that in the previous cycle without MT ( 0 M group), a total of 1838 oocytes were collected, of which 1372 MII oocytes were fertilized by IVF/ICSI, and the fertilization rate was 83.6\% (1147/1372). Among the 140 cycles of 140 patients with subsequent added MT ( $10^{-9} \mathrm{M}$ group), a total of 1658 oocytes were collected, including $1288 \mathrm{MIl}$ oocytes, and the fertilization rate after insemination was $87.7 \%$ (1129/1288). The fertilization, cleavage, high-quality embryo, blastocyst, and high-quality blastocyst rates in the $10^{-9} \mathrm{M}$ group were significantly different compared to the $0 \mathrm{M}$ group $(87.7 \% \mathrm{vs}$. $83.6 \%, p<0.01 ; 94.1 \%$ vs. $90.5 \%, p<0.01 ; 58.3 \%$ vs. $43.8 \%, p<0.0001 ; 51.1 \%$ vs. $41.8 \%, p<0.0001 ; 43.4 \%$ vs. $22.9 \%, p<0.0001)$. 
Table 3

Comparison of oocyte fertilization and embryo development between the $10^{-9} \mathrm{M}$ group and the $0 \mathrm{M}$ group

\begin{tabular}{|c|c|c|}
\hline & $10^{-9} \mathrm{M}$ & $\mathrm{OM}$ \\
\hline Number of cycles & 140 & 240 \\
\hline Number of oocytes & 1658 & 1838 \\
\hline Number of MII & 1288 & 1372 \\
\hline Fertilization rate (\%) & $1129 / 1288(87.7)^{a}$ & 1147/1372(83.6) \\
\hline Cleavage rate $(\%)$ & $1062 / 1129(94.1)^{a}$ & 1038/1147(90.5) \\
\hline High-quality embryo rate (\%) & $619 / 1062(58.3)^{b}$ & $455 / 1038(43.8)$ \\
\hline Blastocyst rate (\%) & $543 / 1062(51.1)^{b}$ & $434 / 1038(41.8)$ \\
\hline High-quality blastocyst rate (\%) & $461 / 1062(43.4)^{b}$ & $238 / 1038(22.9)$ \\
\hline \multicolumn{3}{|c|}{ Data analyzed using chi-squared test or Fisher's exact test. } \\
\hline \multicolumn{3}{|c|}{ Different symbols within columns and different letters within columns and within rov } \\
\hline \multicolumn{3}{|c|}{ indicate significant difference. ${ }^{a} P<0.01$, compared with the 0 MT group; } \\
\hline
\end{tabular}

\section{The result of oocyte fertilization and subsequent embryo development in the $10^{-9} \mathrm{M}$ and $0 \mathrm{M}$ groups of patients who experienced 1 failed IVF/ICSI cycle}

As shown in Fig. 3, there were 1116 oocytes in the $10^{-9} \mathrm{M}$ group and 1009 oocytes in the $0 \mathrm{M}$ group of patients who experienced a single failed IVF/ICSI cycle. In terms of fertilization rate ( $90.1 \%$ vs. $88.2 \%)$ and cleavage rate ( $94.6 \%$ vs. $92.9 \%$ ), the rates of the $10^{-9} \mathrm{M}$ group were higher than the $0 \mathrm{M}$ group, but these differences were not significant. The high-quality embryo rate (56.8\% vs. $39.4 \%)$ and high-quality blastocysts rate ( $42.0 \%$ vs. $25.3 \%$ ) of the $10^{-9} \mathrm{M}$ group was significantly higher than the $0 \mathrm{M}$ group (both $p<0.0001)$.

\section{The result of oocyte fertilization and subsequent embryo development in the $10^{-9} \mathrm{M}$ and $0 \mathrm{M}$ groups of patients who experienced 2 failed IVF/ICSI cycles}


As shown in Fig. 4, there were a total of 468 oocytes in the $10^{-9} \mathrm{M}$ group and 688 oocytes in the $0 \mathrm{M}$ group of patients who experienced two failed IVF/ICSI cycles. The fertilization, cleavage, high-quality embryo and high-quality blastocyst rates of the $10^{-9} \mathrm{M}$ group were higher than those in the $0 \mathrm{M}$ group. The cleavage, high-quality embryo and high-quality blastocyst rates of the $10^{-9} \mathrm{M}$ group were significantly different from those of the $0 \mathrm{M}$ group $(92.2 \%$ vs. $85.6 \%, p<0.05 ; 51.1 \%$ vs. $41.3 \%, p<0.05$, $44.6 \%$ vs. $19.9 \%, p<0.0001)$.

\section{The result of oocyte fertilization and subsequent embryo development in the $10^{-9} \mathrm{M}$ and $0 \mathrm{M}$ groups of patients who experienced $\geq 3$ failed IVF/ICSI cycles}

As shown in Fig. 5, there were a total of 74 oocytes in the $10^{-9} \mathrm{M}$ group and 141 oocytes in the $0 \mathrm{M}$ group of patients who experienced three or more failed IVF/ICSI cycles. The fertilization, cleavage, high-quality embryo and high-quality blastocyst rates of the $10^{-9} \mathrm{M}$ group were higher than those of the $0 \mathrm{M}$ group. Among these, the high-quality embryo and high-quality blastocyst rates of the $10^{-9} \mathrm{M}$ group were significantly different from those of the $0 \mathrm{M}$ group $(45.8 \%$ vs. $34.3 \%, p<0.05,57.1 \%$ vs. $20.9 \%, p<$ 0.0001).

\section{Clinical transplantation outcomes}

By the end of this manuscript submission, the experimental group had conducted 50 cycles of vitrifiedwarmed embryo transfers. A total of 61 warmed blastocysts were transferred, the average number of transfers was 1.22, and 32 cases achieved biochemical pregnancy. The biochemical pregnancy rate was $64 \%$. Subsequently, on the 30-35th day after embryo transfer, B-ultrasound was used to further monitor the condition of the gestational sac, and 20 cases of clinical pregnancy were confirmed. The clinical pregnancy rate was $40 \%$.

\section{Discussion}

In the process of ART treatment, patients encounter many cycles of treatment failure due to poor embryo quality. There are many factors affecting embryo development, such as culture medium [16], $\mathrm{CO}_{2}$ concentration [17], temperature [17], in vitro operations [18, 19], as well as sperm and oocyte quality [20, 21]. It can be considered that during ART treatment, the quality of oocytes is one of the most critical factors that determine the embryo status and clinical outcome. Any factor affecting the quality of the oocyte will affect its fertilization and subsequent embryo development [22], and ultimately the cycle outcome of ART treatment. In this study, an appropriate concentration of MT was added to the embryo culture medium and the embryo development and clinical treatment outcomes of the patients were observed, to understand whether the application of MT has a positive impact on embryo development. 
According to research reports, embryos are in a relatively high oxygen environment during in vitro culture, which will cause oxidative stress due to the imbalance of the antioxidant defense system, resulting in a large number of ROS produced by oocytes and embryos during development, thus affecting the quality of oocytes and outcome of the embryo development in vitro [23]. MT is a highly effective antioxidant with strong antioxidant activity. Animal study has shown that the addition of MT to the embryo culture medium can improve embryo development in vitro, and it has also been confirmed that the improvement is concentration-dependent [4]. In 2020, our team found that adding MT to the human IVM medium can improve the IVM outcome of human immature oocytes by promoting mitochondrial function and inhibiting damage due to oxidative stress, and $10^{-5} \mathrm{M}$ was found to be the optimal concentration [10]. Based on the conclusions of the previous studies, two main questions were posed: (1) can adding MT to the culture medium of the human embryos improve the in vitro development and clinical outcome of the embryos? And (2) is its effect also correlated to concentration? Therefore, in this study, human immature oocytes in the $\mathrm{COH}$ cycle were collected for IVM to obtain IVM-MII oocytes and ICSI insemination was conducted. Next, the fertilized oocytes were cultured in vitro in culture medium containing $0,10^{-11}, 10^{-9}$, $10^{-7}$ or $10^{-5} \mathrm{M} \mathrm{MT}$, and a systematic comparative analysis was performed on the fertilization of oocytes and early embryo development in each group. The fertilization, cleavage, blastocyst and high-quality blastocyst rates of the $10^{-9} \mathrm{M}$ group were all significantly higher than those of the $0 \mathrm{M}$ group. The blastocyst and high-quality blastocyst rates of the $10^{-9} \mathrm{M}$ group were significantly different from those of the $10^{-5} \mathrm{M}$ group, and the blastocyst rate was also significantly different compared to the $10^{-11} \mathrm{M}$ group. In addition, the fertilization, cleavage, blastocyst and high-quality blastocyst rates of the $10^{-9} \mathrm{M}$ group were higher than those of the $10^{-7} \mathrm{M}$ group, but these differences were not significant. It has been reported that the fertilization rate, cleavage rate and total blastocyst cell number of porcine IVF embryos cultured in medium containing $10^{-9} \mathrm{M}$ MT were significantly increased [24]. Wang et al. [4] added different concentrations of MT to the culture medium of bovine embryos in vitro and found that $10^{-9} \mathrm{M}$ MT could significantly promote embryo development. These results are consistent with the results of the present study. These results showed that the addition of $10^{-9} \mathrm{M} \mathrm{MT}$ to human embryo culture medium could significantly improve the fertilization of human oocytes and embryo development in vitro obtained after fertilization, and $10^{-9} \mathrm{M}$ was the optimal concentration.

Next, $10^{-9} \mathrm{M}$ was selected as the MT concentration to be added to the embryo culture medium of patients with failed IVF/ICSI cycles for embryo culture in vitro. The study found that in terms of fertilization, cleavage, high-quality embryo, blastocyst and high-quality blastocyst rates, the values of the $10^{-9} \mathrm{M}$ group were significantly higher than those of the $0 \mathrm{M}$ group. In this study, further group analysis was also carried out according to the number of failed IVF/ICSI cycles and it was discovered that the application of $10^{-9} \mathrm{M}$ MT to embryo culture in vitro could significantly improve embryonic development in the repeated cycle of each group. A large number of studies have found that adding MT to the culture medium can reduce the levels of ROS in oocytes and promote embryonic development of cattle, mice, sheep, and pigs [7, 25-27]. Nakano et al. [28] confirmed that the addition of MT during embryo culture in vitro can reduce the level of ROS in parthenogenetic embryos and promote embryo development. These 
findings are consistent with the results of the current study, suggesting that the improvement of embryonic development in patients with failed IVF/ICSI cycles by MT may be related to the highly effective antioxidant properties of MT. Oxidative stress inhibits oocyte maturation and embryo development, and MT has a strong antioxidant effect, which can resist oxidative stress, maintain the balance of antioxidant system, reduce ROS content in the embryo, and promote gamete maturation and embryo development. In this study, 50 cycles of vitrified-warmed embryo transfers with MT intervention have been performed, and a biochemical pregnancy rate of $64 \%$ was achieved, as well as a clinical pregnancy rate of $40 \%$. This result shows that the application of $10^{-9} \mathrm{M} \mathrm{MT}$ to embryo culture medium in vitro can significantly improve the clinical treatment effect of patients with failed IVF/ICSI cycles, which greatly enhance our confidence in treating such patients, and thereby increases the overall success rate of ART treatment.

In conclusion, the application of MT to embryo culture medium in vitro can improve embryonic development for patients with repeated cycles after failed IVF/ICSI cycles and lead to good clinical outcomes. The optimal concentration of MT was $10^{-9} \mathrm{M}$.

\section{Abbreviations}

MT: melatonin; IVF: In vitro fertilization; ICSI: Intracytoplasmic sperm injection; IVM: In vitro maturation; ART: Assisted reproductive technology; IVF-ET: In vitro fertilization and embryo transfer; ROS: Reactive oxygen species; GSH: glutathione; $\mathrm{COH}$ : Controlled ovarian hyperstimulation; $\mathrm{GnRH}$ : Gonadotropin hormone-releasing hormone; HCG: Human chorionic gonadotropin; GnRH-A: GnRH antagonist; COC: Cumulus-oocyte complex; FSH: Follicle-stimulating hormone; LH: Luteinizing hormone; BMI: Body mass index.

\section{Declarations}

\section{Ethics approval and consent to participate}

This study was approved by the biomedical ethics committee of Anhui Medical University (2015013).

\section{Consent for publication}

All authors provided final approval of the version to be published and agree to be accountable for all aspects of the work in ensuring that questions.

\section{Availability of data and materials}

The datasets used and/or analyzed during the current study are available from the corresponding author on reasonable request.

\section{Competing interests}


The authors declare that there are no conflicts of interest.

\section{Funding}

This work was supported by National Key Technology R\&D Program of China (No. 2017YFC1002004), General project of National Natural Science Foundation of China: molecular mechanism of melatonin regulating freezing injury of human oocytes during vitrification(82071724), National Major Scientific Research Instrument and Equipment Development Project (11627803) and Open project of State Key Laboratory of reproductive medicine: mechanism of melatonin promoting maturation of immature oocytes and subsequent embryo development by protecting mitochondrial membrane dynamics(SKLRMK202005).

\section{Authors' contributions}

Qi Zhu: Manuscript writing and sample collection; Kaijuan Wang and Chao Zhang: Data collection; Zhaojuan Yu and Bihua Rao: Data analysis; Beili Chen, Huijuan Zou and Weiwei Zou: clinical assessments and experiment conducting; Yunxia Cao: Manuscript editing; Ding Ding and Zhiguo Zhang: Manuscript revising and study design. All authors read and approved the final manuscript.

\section{Acknowledgements}

We gratefully acknowledge all the participants in this study.

\section{References}

1. Esteves SC, Humaidan P, Roque M, Agarwal A. Female infertility and assisted reproductive technology. Panminerva Med. 2019;61:1-2.

2. Liu J, Zhang X, Yang Y, Zhao J, Hao D, Zhang J, Liu Y, Wu W, Wang X. Long-time vs. short-time insemination of sibling eggs. Exp Ther Med. 2016;12:3756-60.

3. M OJ. Melatonin and Female Reproduction: An Expanding Universe. Front Endocrinol. 2020;11:85.

4. Wang F, Tian X, Zhang L, Gao C, He C, Fu Y, Ji P, Li Y, Li N, Liu G. Beneficial effects of melatonin on in vitro bovine embryonic development are mediated by melatonin receptor 1 . J Pineal Res. 2014;56:333-42.

5. Manchester LC, Coto-Montes A, Boga JA, Andersen LPH, Zhou Z, Galano A, Vriend J, Tan D, Reiter RJ. Melatonin: an ancient molecule that makes oxygen metabolically tolerable. J Pineal Res. 2015;59:403-19.

6. An Q, Peng W, Cheng Y, Lu Z, Zhou C, Zhang Y, Su J. Melatonin supplementation during in vitro maturation of oocyte enhances subsequent development of bovine cloned embryos. J Cell Physiol. 2019;234:17370-81.

7. Barros VRP, Monte APO, Santos JMS, Lins TLBG, Cavalcante AYP, Gouveia BB, Müller MC, Oliveira Junior JL, Barberino RS, Donfack NJ, Araújo VR, Matos MHT. Effects of melatonin on the in vitro 
growth of early antral follicles and maturation of ovine oocytes. Domest Anim Endocrin. 2020;71:106386.

8. Lin T, Lee JE, Kang JW, Oqani RK, Cho ES, Kim SB, II Jin D. Melatonin supplementation during prolonged in vitro maturation improves the quality and development of poor-quality porcine oocytes via anti-oxidative and anti-apoptotic effects. Mol Reprod Dev. 2018;85:665-81.

9. Li X, Mu Y, Elshewy N, Ding D, Zou H, Chen B, Chen C, Wei Z, Cao Y, Zhou P, Zhang Z. Comparison of IVF and IVM outcomes in the same patient treated with a modified IVM protocol along with an oocytes-maturing system containing melatonin: A pilot study. Life Sci. 2021;264:118706.

10. Zou H, Chen B, Ding D, Gao M, Chen D, Liu Y, Hao Y, Zou W, Ji D, Zhou P, Wei Z, Cao Y, Zhang Z. Melatonin promotes the development of immature oocytes from the $\mathrm{COH}$ cycle into healthy offspring by protecting mitochondrial function. J Pineal Res. 2019;68(1):e12621.

11. Sacha CR, Kaser DJ, Farland LV, Srouji S, Missmer SA, Racowsky C. The effect of short-term exposure of cumulus-oocyte complexes to in vitro maturation medium on yield of mature oocytes and usable embryos in stimulated cycles. J Assist Reprod Gen. 2018;35:841-9.

12. Ding D, Wang Q, Li X, Chen B, Zou W, Ji D, Hao Y, Xue R, Zou H, Wei Z, Zhou P, Cao Y, Zhang Z. Effects of different polyvinylpyrrolidone concentrations on intracytoplasmic sperm injection. Zygote. 2020;28:148-53.

13. Tomás $C$, Orava $M$, Tuomivaara $L$, Martikainen $H$. Low pregnancy rate is achieved in patients treated with intracytoplasmic sperm injection due to previous low or failed fertilization in in-vitro fertilization. Human reproduction (Oxford). 1998;13:65-70.

14. Gardner DK, Schoolcraft WB. Culture and transfer of human blastocysts. Curr Opin Obstet Gynecol. 1999;11:307-11.

15. Maria GRS, Camargos JKRV, Monique EG, Nunes AFCF. Human oocyte morphometry before and after cryopreservation: A prospective cohort study. Cryobiology. 2019;88:81-6.

16. Tarahomi M, Vaz FM, Straalen JPV, Schrauwen FAP, Wely MV, Hamer G, Repping S, Mastenbroek S. The composition of human preimplantation embryo culture media and their stability during storage and culture. Hum Reprod. 2019;34:1450-61.

17. Brom-De-Luna JG, Salgado RM, Canesin HS, Diaw M, Hinrichs K. Equine blastocyst production under different incubation temperatures and different CO2 concentrations during early cleavage. Reproduction Fertility Development. 2019;31:1823-9.

18. Imesch P, Scheiner D, Xie M, Fink D, Macas E, Dubey R, Imthurn B. Developmental potential of human oocytes matured in vitro followed by vitrification and activation. J Ovarian Res. 2013;6:30.

19. Lu X, Liu Y, Cao X, Liu S, Dong X. Laser-assisted hatching and clinical outcomes in frozen-thawed cleavage-embryo transfers of patients with previous repeated failure. Laser Med Sci. 2019;34:113745.

20. Ribas-Maynou J, Benet J. Single and Double Strand Sperm DNA Damage: Different Reproductive Effects on Male Fertility. Genes-Basel. 2019;10:105. 
21. Keefe D, Kumar M, Kalmbach K. Oocyte competency is the key to embryo potential. Fertil Steril. 2015;103:317-22.

22. Miyara F. Multiparameter analysis of human oocytes at metaphase II stage after IVF failure in nonmale infertility. Hum Reprod. 2003;18:1494-503.

23. Li C, Hao H, Zhao Y, Zhang P, Wang H, Pang Y, Du W, Zhao S, Liu Y, Huang J, Wang J, Ruan W, Hao T, Reiter RJ, Zhu H, Zhao X. Melatonin Improves the Fertilization Capacity of Sex-Sorted Bull Sperm by Inhibiting Apoptosis and Increasing Fertilization Capacitation via MT1. Int J Mol Sci. 2019;20:3921.

24. Lord T, Nixon B, Jones KT, Aitken RJ. Melatonin Prevents Postovulatory Oocyte Aging in the Mouse and Extends the Window for Optimal Fertilization In Vitro1. Biol Reprod. 2013;88:67.

25. Kang JT, Koo OJ, Kwon DK, Park HJ, Jang G, Kang SK, Lee BC. Effects of melatonin on in vitro maturation of porcine oocyte and expression of melatonin receptor RNA in cumulus and granulosa cells. J Pineal Res. 2009;46:22-8.

26. Barros VRP, Monte APO, Santos JMS, Lins TLBG, Cavalcante AYP, Gouveia BB, Müller MC, Oliveira JL, Donfack NJ, Araújo VR, Matos MHT. Melatonin improves development, mitochondrial function and promotes the meiotic resumption of sheep oocytes from in vitro grown secondary follicles. Theriogenology. 2020;144:67-73.

27. Zhao X, Hao H, Du W, Zhao S, Wang H, Wang N, Wang D, Liu Y, Qin T, Zhu H. Melatonin inhibits apoptosis and improves the developmental potential of vitrified bovine oocytes. J Pineal Res. 2016;60:132-41.

28. Nakano M, Kato Y, Tsunoda Y. Effect of melatonin treatment on the developmental potential of parthenogenetic and somatic cell nuclear-transferred porcine oocytesin vitro. Zygote. 2012;20:199207.

\section{Figures}




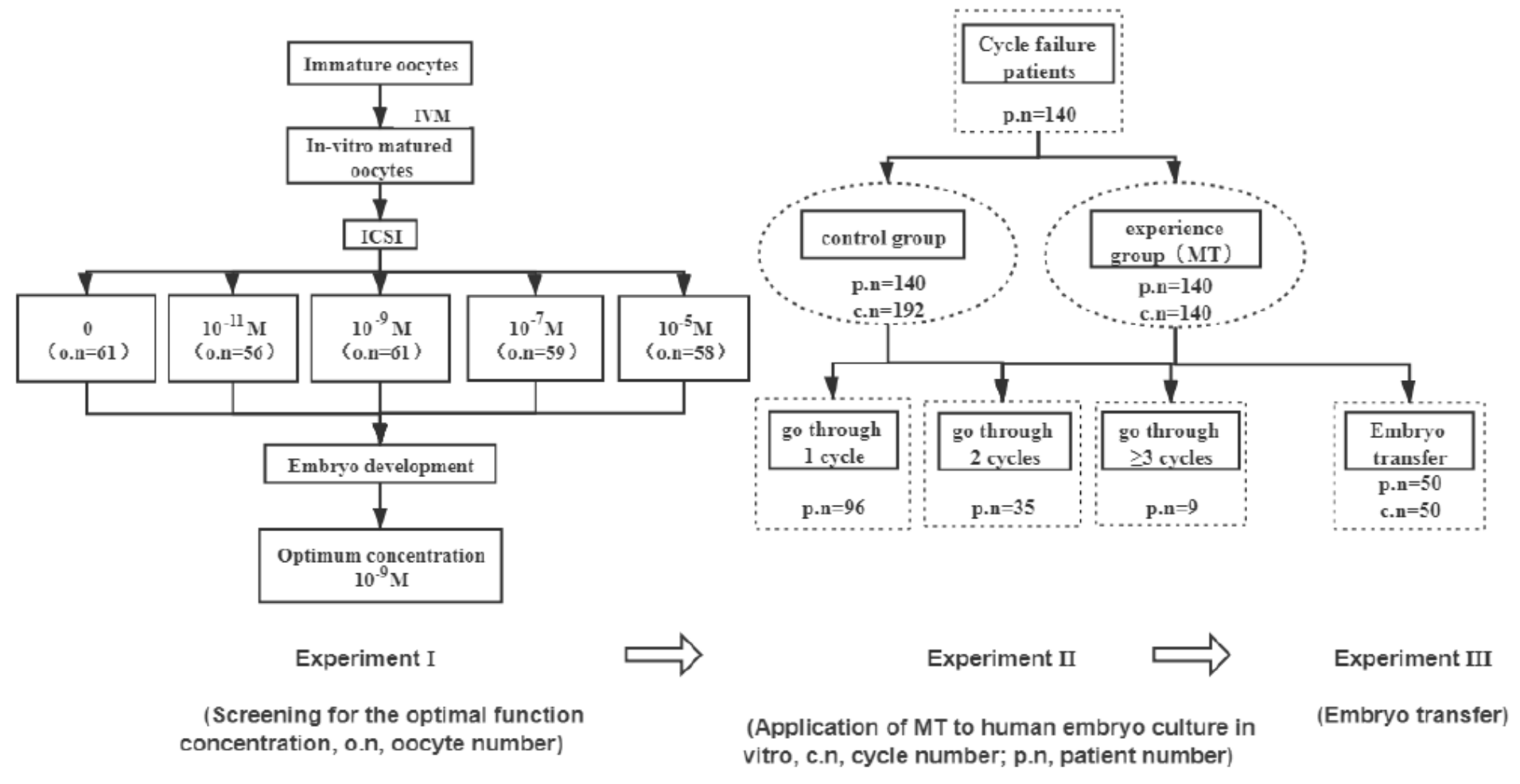

Figure 1

Experimental flow chart. o.n, oocyte number; c.n, cycle number; p.n, patient number.
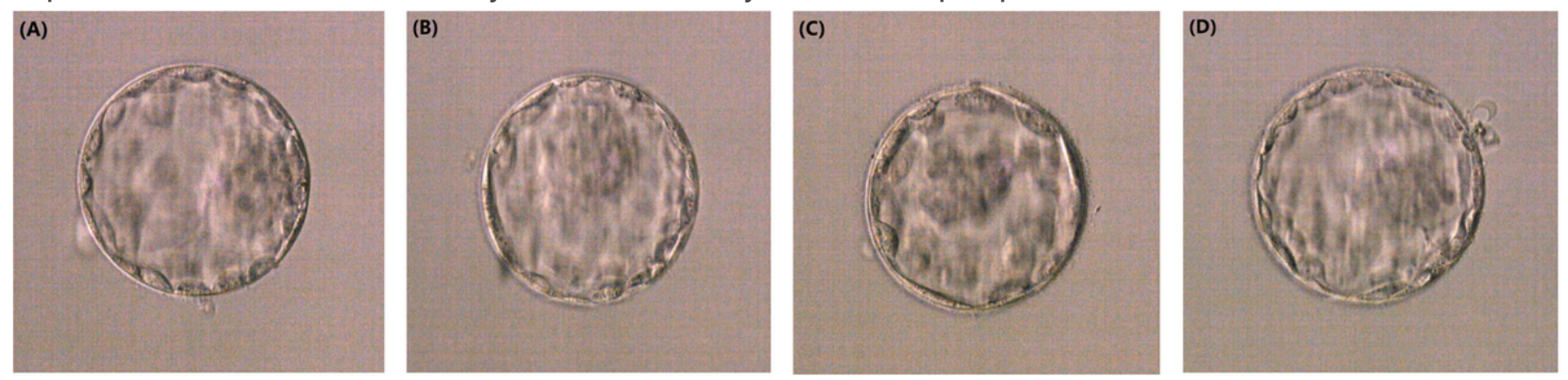

\section{Figure 2}

Representative images of the high-quality blastocysts. Note: (A) High-quality blastocyst 4AA; (B) highquality blastocyst 4AA; (C) high-quality blastocyst 4AB; (D) high-quality blastocyst 4BA. Scale bar=10uM. 
A

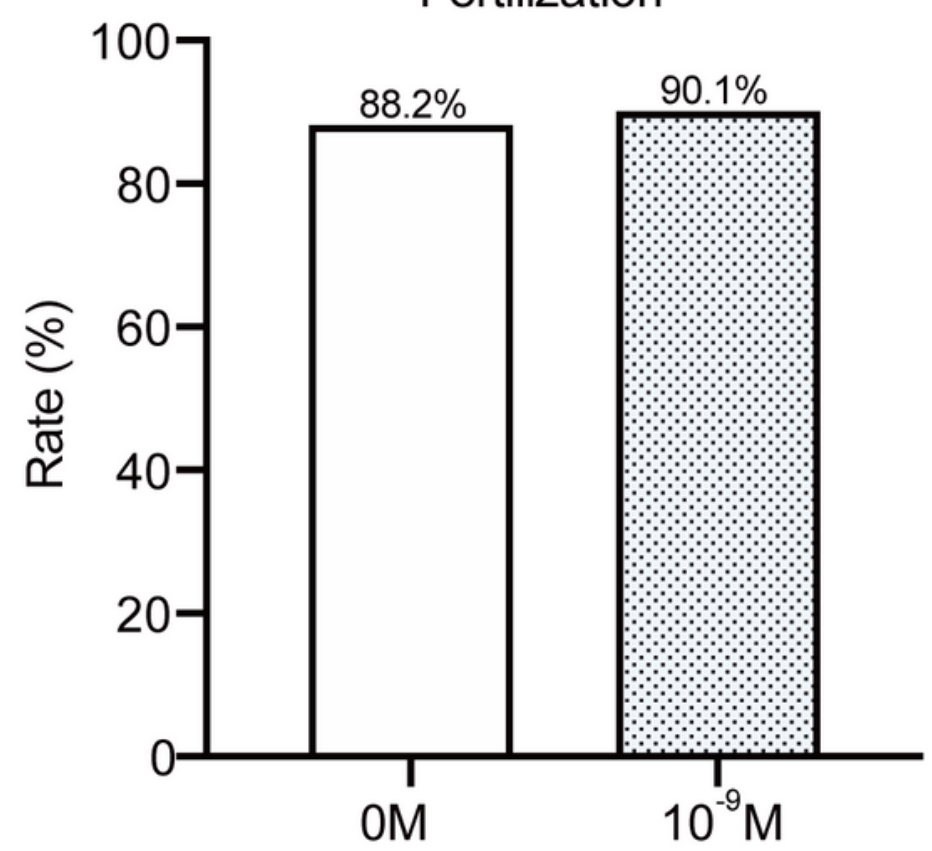

C High-quality cleavage embryo

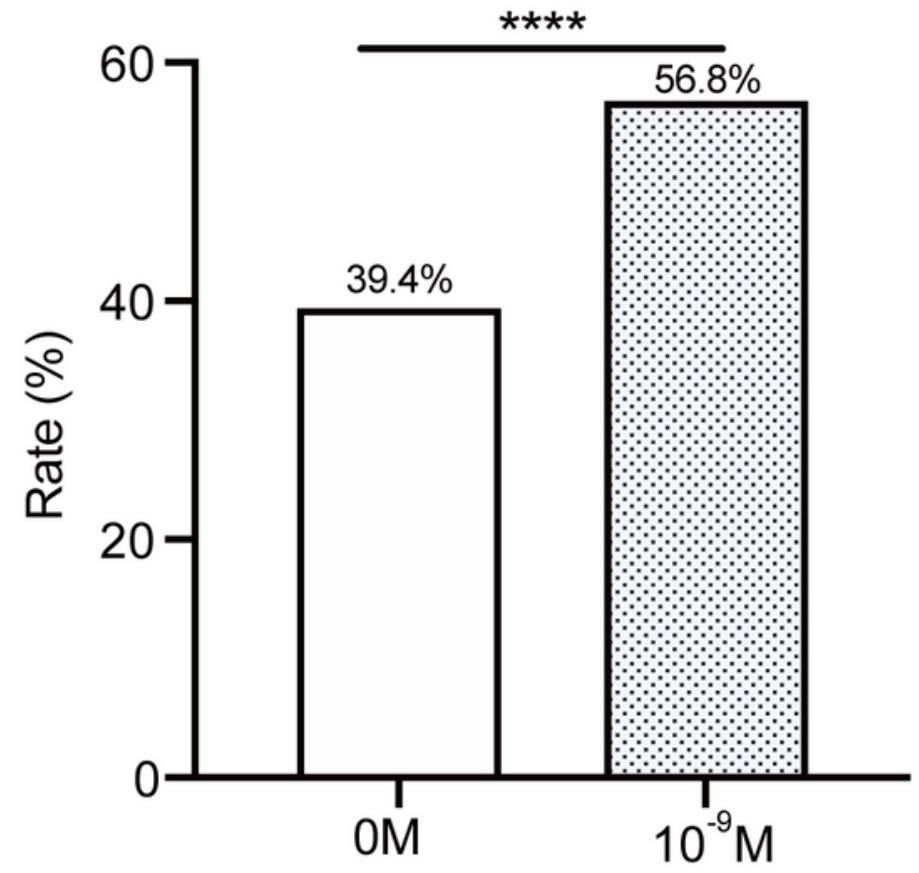

B

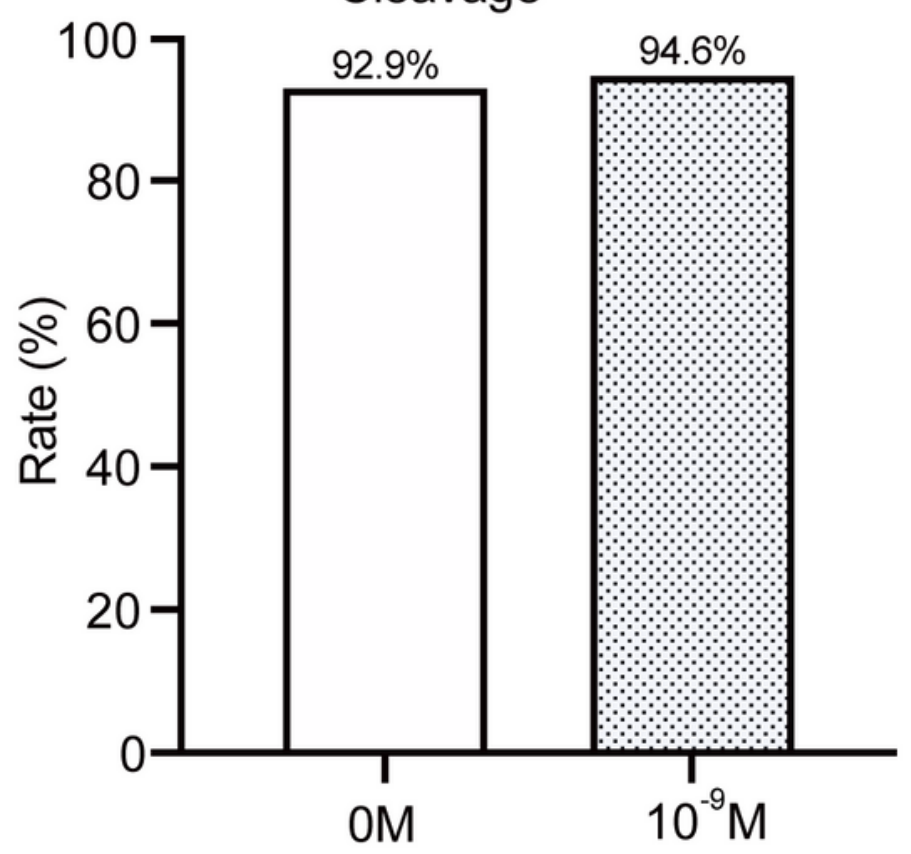

D

High-quality blastocyst

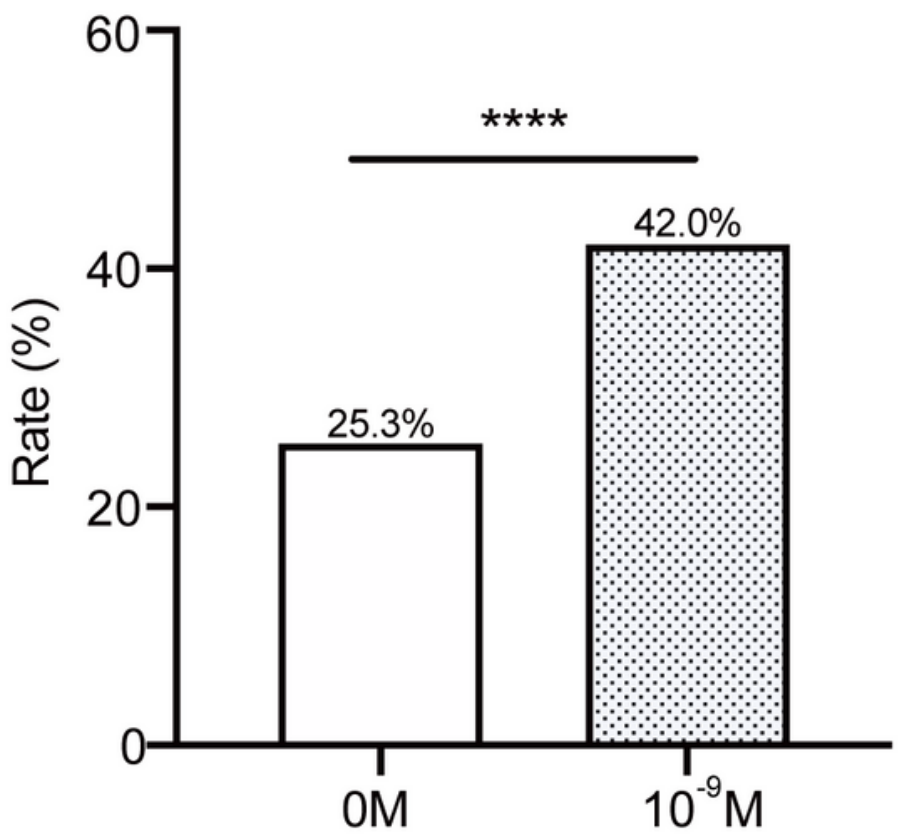

Figure 3

Status of fertilization and early embryo development in patients with one failed IVF/ICSI cycle. Note: A: fertilization; B: cleavage; C: high-quality embryo and D: high-quality blastocyst. ( $\left.{ }^{\star} \star \star * P<0.0001\right)$. 

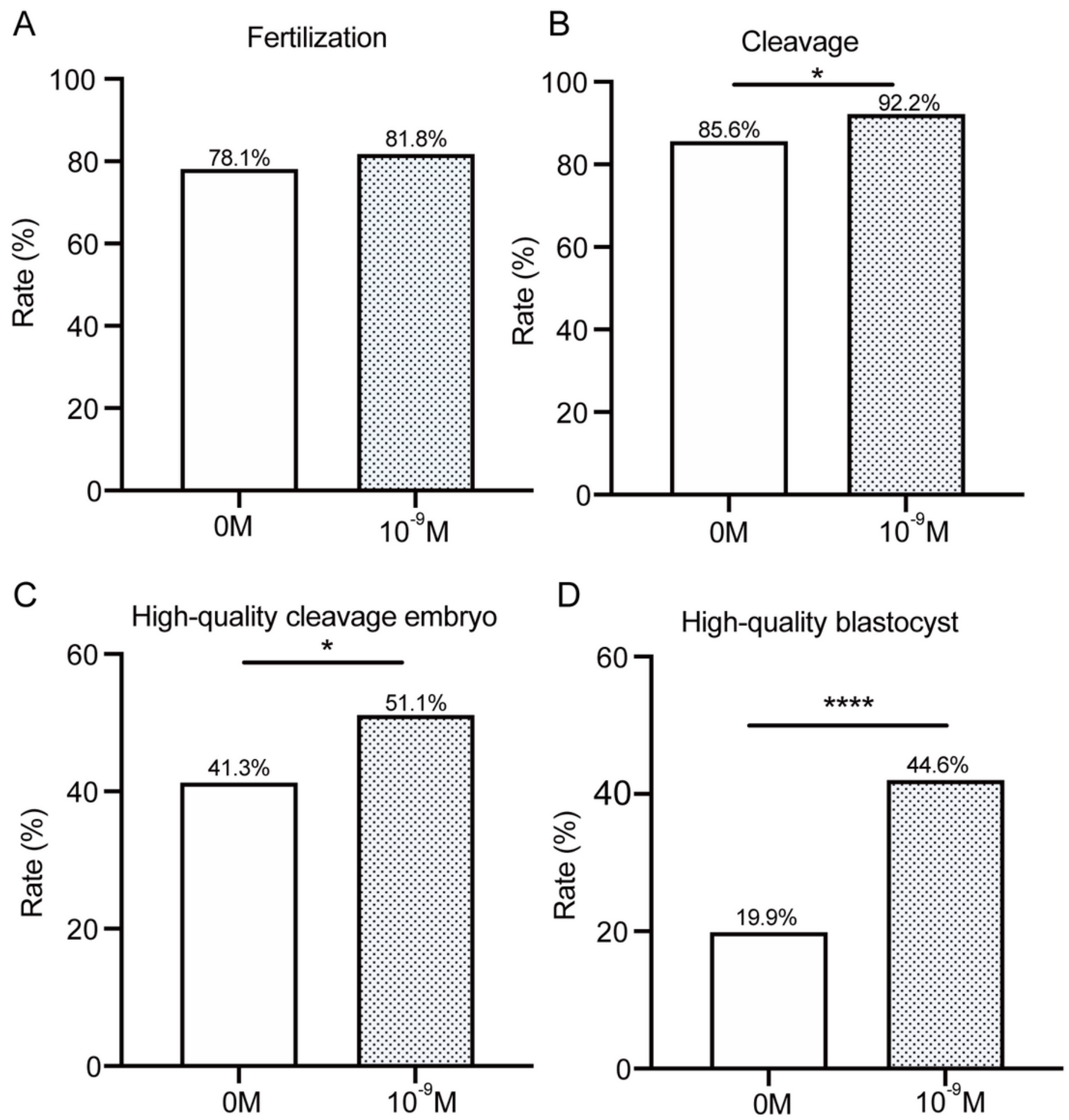

Figure 4

Status of fertilization and early embryo development in patients with two failed IVF/ICSI cycles. Note: A: fertilization; B: cleavage; C: high-quality embryo and D: high-quality blastocyst. ( $\left.{ }^{\star} P<0.05 ; \star \star \star \star P<0.0001\right)$. 

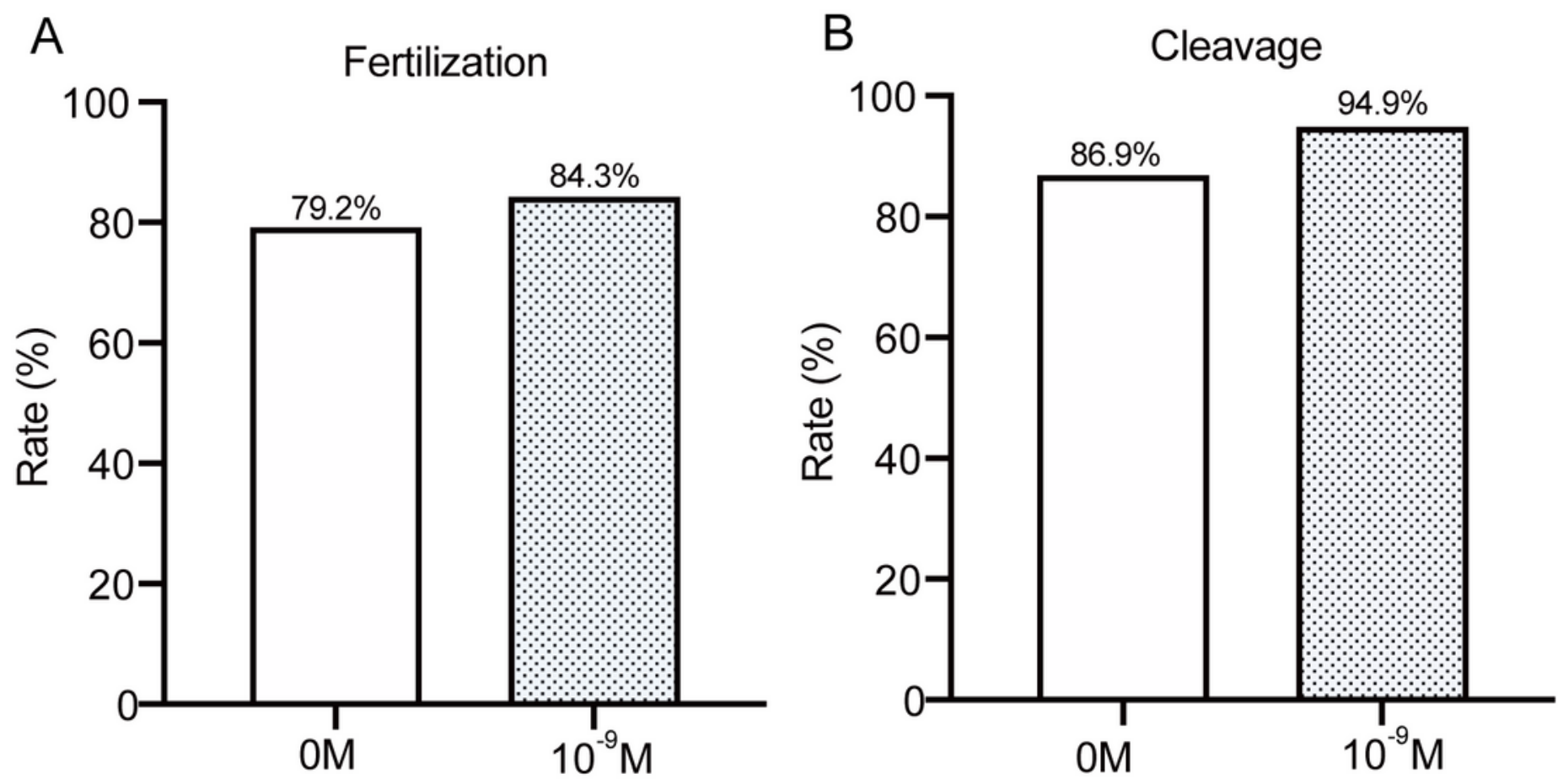

C High-quality cleavage embryo

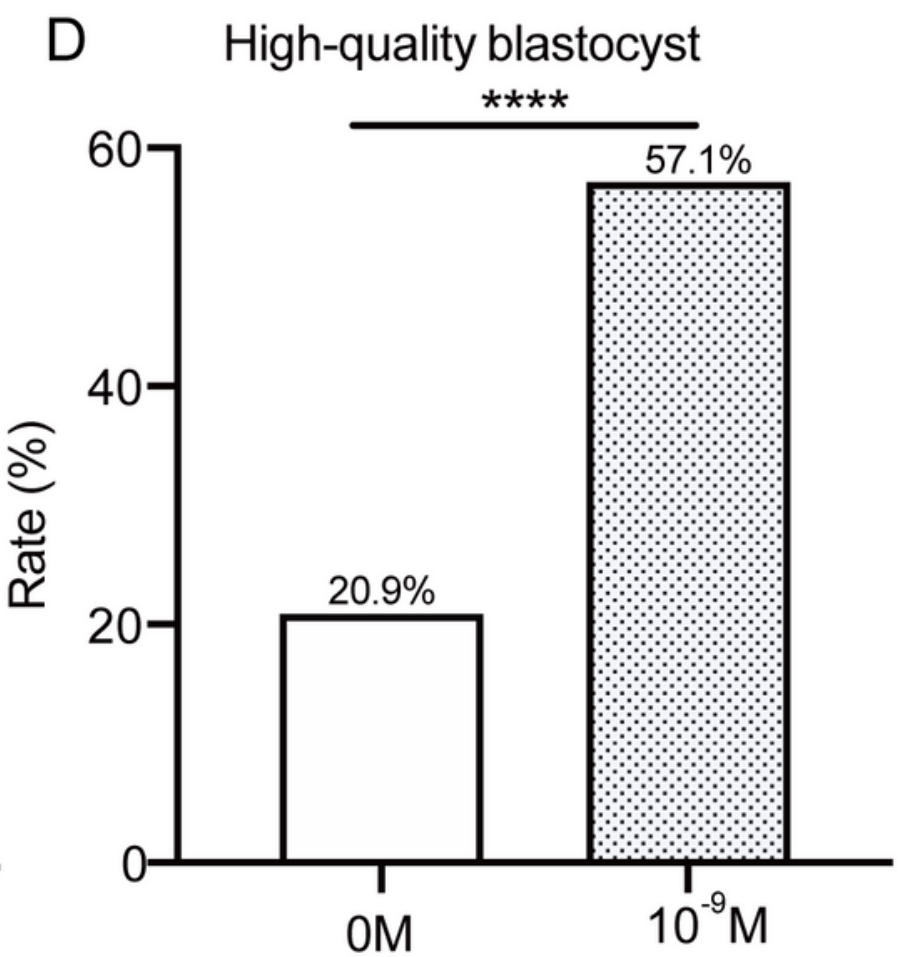

Figure 5

Status of fertilization and early embryo development in patients with one failed IVF/ICSI cycles. Note: A: fertilization; B: cleavage; C: high-quality embryo and $D$ : high-quality blastocyst. $\left.{ }^{\star} P<0.05 ; * \star \star \star P<0.0001\right)$. 